\title{
A Wave Near the Edge of a Circular Disk
}

\author{
M. Destrade ${ }^{*, a}$ and Y.B. Fu \\ ${ }^{a}$ Institut Jean Le Rond d'Alembert, CNRS, Université Pierre et Marie Curie, France \\ ${ }^{b}$ Department of Mathematics, Keele University, UK
}

\begin{abstract}
It is shown that in the Love-Kirchhoff plate theory, an edge wave can travel in a circular thin disk made of an isotropic elastic material. This disk edge wave turns out to be faster than the classic flexural acoustic wave in a straightedged, semi-infinite, thin plate, a wave which it mimics when the curvature radius becomes very large compared to the wavelength.
\end{abstract}

\section{INTRODUCTION}

When an elastic half-space is subjected to a point excitation, it responds by generating waves which become surface (Rayleigh) waves in the far-field. When a cymbal is hit on its edge, it produces a long-lasting sound: why not conjecture that this phenomenon is due to the propagation of an acoustic wave localized near the edge of the cymbal?

The study of vibrations of thin circular plates with free edges dates back to at least 1910 with a paper by Airey [1], and is still a very active area of research (see for instance Touzé, Thomas, and Chaigne [2]). In contrast there seems to be almost no contribution on the subject of waves in these plates, whereas there exist a wealth of papers on waves over the surface of cylinders with infinite extent. That scarcity might be explained as follows. In the latter case (cylinder), the asymptotic behavior of a wave when the radius of the cylinder becomes very large (in comparison to the wavelength) is well-known: it is that of the celebrated Rayleigh wave in a half-space [3]. In the former case (disk), the asymptotic behavior is not as well-known: it is that of a wave propagating along the straight, free edge of a thin Kirchhoff plate [4]. Hence Cerv [5] studied waves traveling on the surface of a thin disk, but used plane-stress equations, where the thickness of the plate does not appear: as a result the asymptotic behavior of his waves is that of Rayleigh waves.

This paper shows that the appropriate resolution of the classical small-deflection equations of motion in a circular disk causes no particular problem. It is conducted with a view to interpret some patterns of motion observed near the edge of a stricken cymbal [6] (other experimental results are found in [7]).

Other related problems are left open but should pose no particular difficulty to solve; they include the following. (i) The complimentary problem of acoustic waves propagating along the edge of a circular hole in a thin plate, with obvious possible applications in non-destructive evaluation, see Fig. (1). (ii) A circular disk made of one material in rigid or slippery contact with a holed plate made of another material;

*Address correspondence to this author at the Institut Jean Le Rond d'Alembert, CNRS, Université Pierre et Marie Curie, Boite 162, 4 Place Jussieu, 75252 Paris Cedex 05, France; E-mail: destrade@1mm.jussieu.fr there the asymptotic behavior is that of contact flexural waves in thin plates [8] and the applications are also in the realm of non-destructive evaluation (of the strength of rivets for example). (iii) Waves traveling in a thin flat annulus; there the asymptotic behavior is that of flexural waves on a narrow plate [9] and an exotic application would be the transportation of planar heavy objects by acoustic levitation [10] on circular transporting systems. (iv) Another possibility resides in linking the present results to the experimental work of Shaw [11] on Barium Titanate disks.

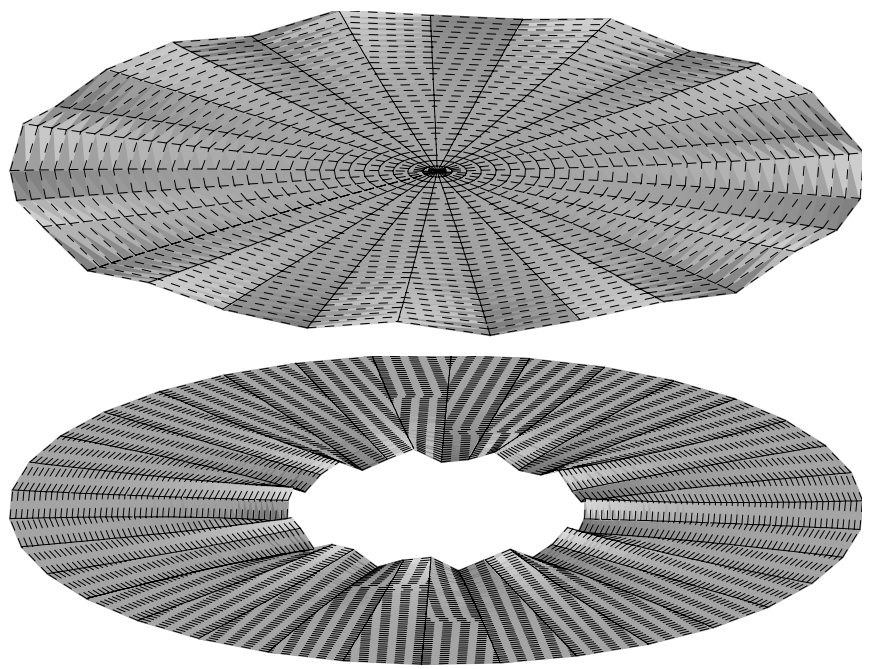

Fig. (1). Wave localized near the edge of a disk or a hole.

\section{BASIC EQUATIONS}

The equations of motion for a Love-Kirchhoff plate are [12]:

$\left(V_{s} h\right)^{2} \nabla^{4} w+6(1-v) \ddot{w}=0$,

where $w$ is the deflection, $h$ is the thickness of the plate, $v$ its Poisson ratio, and $V_{s}$ its shear wave speed. Now consider an acoustic flexural wave traveling along the circumference of a circle (axis normal to the plate, radius $R$ ) in the form

$w=W(r) \cos (k R \theta-\omega t)$,

where $r, \theta$ are the in-plane cylindrical coordinates, $k$ is the wavenumber, and $\omega$ the frequency. Introducing the dimensionless "speed" $\alpha$, defined by [13]. 
$\alpha^{2}=6(1-v)\left(\frac{V}{V_{s}}\right)^{4}\left(\frac{V_{s}}{\omega h}\right)^{2}$, where $\quad V:=\frac{\omega}{k}$,

The equations of motion can be put in the form

$D_{+} D_{-} W=D_{-} D_{+} W=0$,

where $D_{ \pm}$are the following differential operators:

$D_{ \pm}:=d^{2} / d r^{2}+(1 / r) d / d r-k^{2} R^{2} / r^{2} \pm \alpha k^{2}$.

Clearly, the general solution to this differential equation is a linear combination of Bessel functions; specifically,

$$
\begin{aligned}
& W(r)=\gamma_{1} J_{p}(\sqrt{\alpha} k r)+\gamma_{2} I_{p}(\sqrt{\alpha} k r) \\
& +\gamma_{3} Y_{p}(\sqrt{\alpha} k r)+\gamma_{4} K_{p}(\sqrt{\alpha} k r), \quad p:=k R .
\end{aligned}
$$

Here, the $\gamma_{i}$ are constants and the labeling of Bessel functions and modified Bessel functions follows the conventions of Abramowitz and Stegun [14]. In particular,

$$
\begin{aligned}
& D_{+} J_{p}(\sqrt{\alpha} k r)=D_{+} Y_{p}(\sqrt{\alpha} k r) \\
& =D_{-} I_{p}(\sqrt{\alpha} k r)=D_{-} K_{p}(\sqrt{\alpha} k r)=0 .
\end{aligned}
$$

Then for the edge of the circle to be free, two boundary conditions must be satisfied [12]. First, the edge shear must vanish, so that

$$
\frac{\partial^{2} w}{\partial r^{2}}+v\left(\frac{1}{r} \frac{\partial w}{\partial r}+\frac{1}{r^{2}} \frac{\partial^{2} w}{\partial \theta^{2}}\right)=0 \quad \text { at } r=R
$$

Second, the rate of change of the twisting moment must also vanish, so that

$$
\frac{\partial}{\partial r}\left(\nabla^{2} w\right)+\frac{(1-v)}{r} \frac{\partial}{\partial \theta}\left(\frac{1}{r} \frac{\partial^{2} w}{\partial r \partial \theta}-\frac{1}{r^{2}} \frac{\partial w}{\partial \theta}\right)=0
$$

at $r=R$.

Finally, the displacement at $\theta$ is the displacement at $\theta+2 \pi, \theta+4 \pi$, etc., and so by (2), $p=k R$ must be an integer. In other words, the wave can only travel under the condition that its wavelength is an integer fraction of the circle perimeter.

\section{CIRCULAR DISK}

For a wave on the edge of a circular disk, take $\gamma_{3}=\gamma_{4}=0$ in (6) to avoid singularities at the center of the disk. Then the boundary conditions (8) and (9) reduce, after much manipulation and use of (7), to

$$
\begin{aligned}
& J_{p}(\sqrt{\alpha} p)\left[(1-v) j_{p}(\alpha) / p-(1-v-\alpha)\right] v_{1} \\
& +I_{p}(\sqrt{\alpha} p)\left[(1-v) i_{p}(\alpha) / p-(1-v+\alpha)\right] v_{2}=0
\end{aligned}
$$

and

$$
\begin{aligned}
& J_{p}(\sqrt{\alpha} p)\left[(1-v+\alpha) j_{p}(\alpha)-(1-v) / p\right] v_{1} \\
& +I_{p}(\sqrt{\alpha} p)\left[(1-v-\alpha) i_{p}(\alpha)-(1-v) / p\right] v_{2}=0
\end{aligned}
$$

respectively. Here the functions $j_{p}(\alpha)$ and $i_{p}(\alpha)$ are defined by $j_{p}(\alpha):=\sqrt{\alpha} \frac{J_{p}(\sqrt{\alpha} p)}{J_{p}(\sqrt{\alpha} p)} \sim \sqrt{1-\alpha} \quad$ as $p \rightarrow \infty$,

$i_{p}(\alpha):=\sqrt{\alpha} \frac{I_{p}(\sqrt{\alpha} p)}{I_{p}(\sqrt{\alpha} p)} \sim \sqrt{1+\alpha} \quad$ as $p \rightarrow \infty$,

and their asymptotic behavior as $p$ becomes large is found from Debye series expansions [14]. For non-trivial solutions to exist, the following dispersion equation must be satisfied:

$(1-v-\alpha)^{2} i_{p}(\alpha)-(1-v+\alpha)^{2} j_{p}(\alpha)$

$+2 \alpha\left[1+i_{p}(\alpha) j_{p}(\alpha)\right](1-v) / p$

$-\left[i_{p}(\alpha)-j_{p}(\alpha)\right](1-v)^{2} / p^{2}=0$.

Not surprisingly, the dispersion equation for disk edge waves is the same as the frequency equation $[1,16]$ derived for the vibrations of a circular disk with free edges, simply because the governing equations and the boundary conditions happen to coincide in the present context for waves such as (2) and for vibrations in the form $w=W(r) \cos (k R \theta) \cos (\omega t)$, say. The interpretation is however different. In particular, when the radius of the disk becomes large with respect to the wavelength, $p \rightarrow \infty$ and this equation becomes that of the edge wave on a semi-infinite plate discovered by Konenkov [4] and hereafter called the "straight-edge wave",

$(1-v-\alpha)^{2} \sqrt{1+\alpha}=(1-v+\alpha)^{2} \sqrt{1-\alpha}$.

Note that in general, the dispersion equation (13) gives several roots, numbered by an integer parameter $s$, say. The smallest root $(s=0)$ corresponds to a solution which is never zero over the surface of the disk; the next root $(s=1)$ corresponds to a solution which is zero once over the surface of the disk, and so on. Hence $s$ denotes the number of nodal circles [16].

Table 1 displays the values of $\alpha$ for various Poisson ratios and integer values of $p$ at $s=0$; clearly, $\alpha$ tends to the straight-edge wave speed as $p \rightarrow \infty$ (last column, see also Thurston and McKenna [13]); also, the disk edge wave is always faster than the straight-edge wave (note that similarly, the wave propagating on the free surface of a cylinder is faster than the Rayleigh wave propagating over the free surface of an elastic half-space [15].) For the one-mode $(p=1)$ wave, $\alpha$ is nearly twice its value for the straightedge wave $(p \rightarrow \infty)$ and then it decreases rapidly as $p$ grows. Multiplying the values displayed by $p^{2}$ gives the quantity computed and collected by Leissa [16]; the reader is also referred to that book for values when $s \geq 1$.

For the purpose of comparison, recall that Viktorov [15] studied waves over the convex surface of a full cylinder, plotted their variation with depth for a cylinder made of brass, at $p=5,41, \infty$, and found that they "decay somewhat more rapidly with distance from the surface than for a Rayleigh wave, the rate of decay being faster for smaller $p=k R$ ". Fig. (2) shows that the depth profiles (normalized 
with respect to the wavelength) at $p=5,41,200, \infty$ and $s=0$ for a brass disk follow a similar trend. Note however that the edge waves on a disk decay much more rapidly than the straight-edge wave, which is known to be weakly inhomogeneous [17].

Table 1. Values of $\alpha$ for Various $v$ and $p(\mathrm{~s}=0)$

\begin{tabular}{|c|c|c|c|c|c|}
\hline $\mathbf{v}^{\boldsymbol{p}}$ & $\mathbf{5}$ & $\mathbf{1 0}$ & $\mathbf{5 0}$ & $\mathbf{1 0 0}$ & $\boldsymbol{\infty}$ \\
\hline \hline 0.1 & 1.4312 & 1.3054 & 1.1135 & 1.0722 & 0.99998 \\
\hline 0.17 & 1.4035 & 1.2886 & 1.1081 & 1.0686 & 0.99985 \\
\hline 0.2 & 1.3903 & 1.2803 & 1.1051 & 1.0666 & 0.99970 \\
\hline 0.25 & 1.3664 & 1.2646 & 1.0991 & 1.0623 & 0.99918 \\
\hline 0.3 & 1.3398 & 1.2465 & 1.0914 & 1.0567 & 0.99810 \\
\hline $1 / 3$ & 1.3205 & 1.2330 & 1.0853 & 1.0521 & 0.99690 \\
\hline 0.4 & 1.2776 & 1.2010 & 1.0701 & 1.0403 & 0.99264 \\
\hline
\end{tabular}

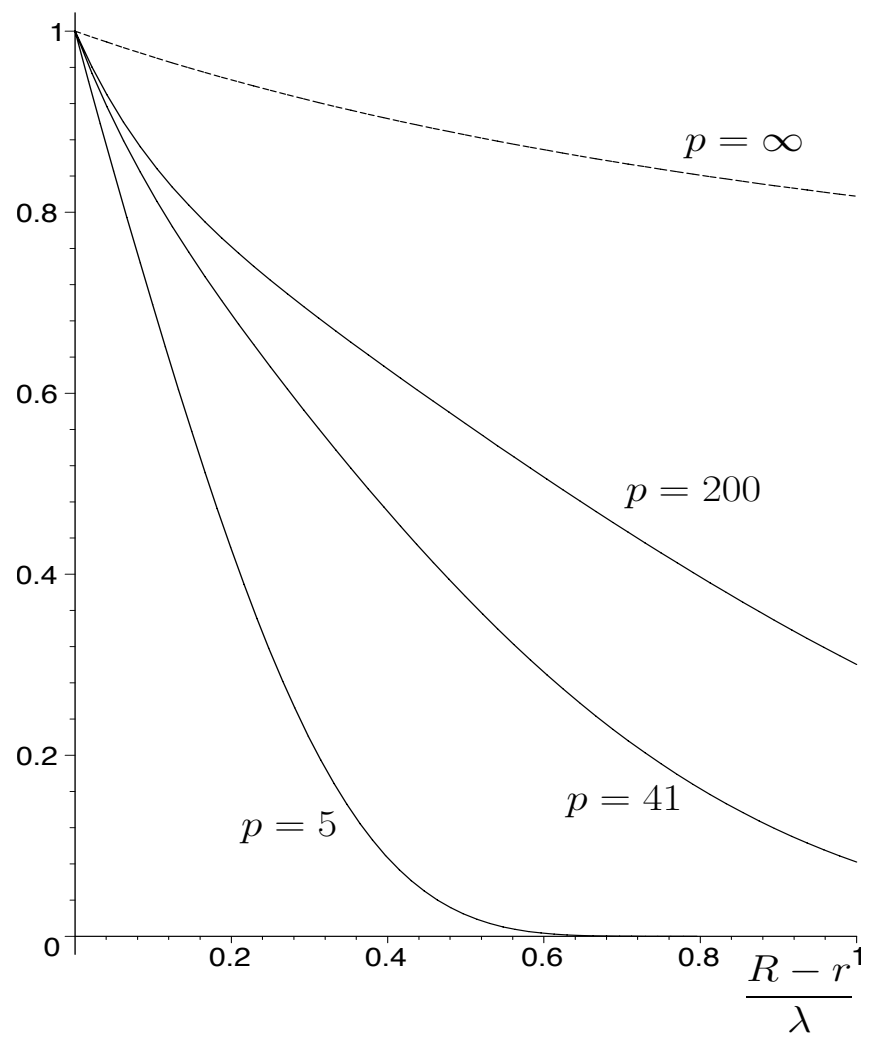

Fig. (2). Depth profiles for a thin disk made of brass $(v=0.34)$.

Schedin, Gren, and Rossing [6] used double-pulsed television holography to record the response of a cymbal made of brass $(v=0.34)$ when it is struck near its edge. They noted that "high-frequency waves are apparent along the edge" and these can be clearly seen on their pictures. A rough estimate gives a number of about 12 wavelengths in a $\pi R / 8$ sector, indicating that $p$ is about 200. Fig. (3) displays the dispersion curves for brass for $s=0,1,2,3$, up to $p=200$. Here, the discrete nature of the plots has been overridden for ease of reading.

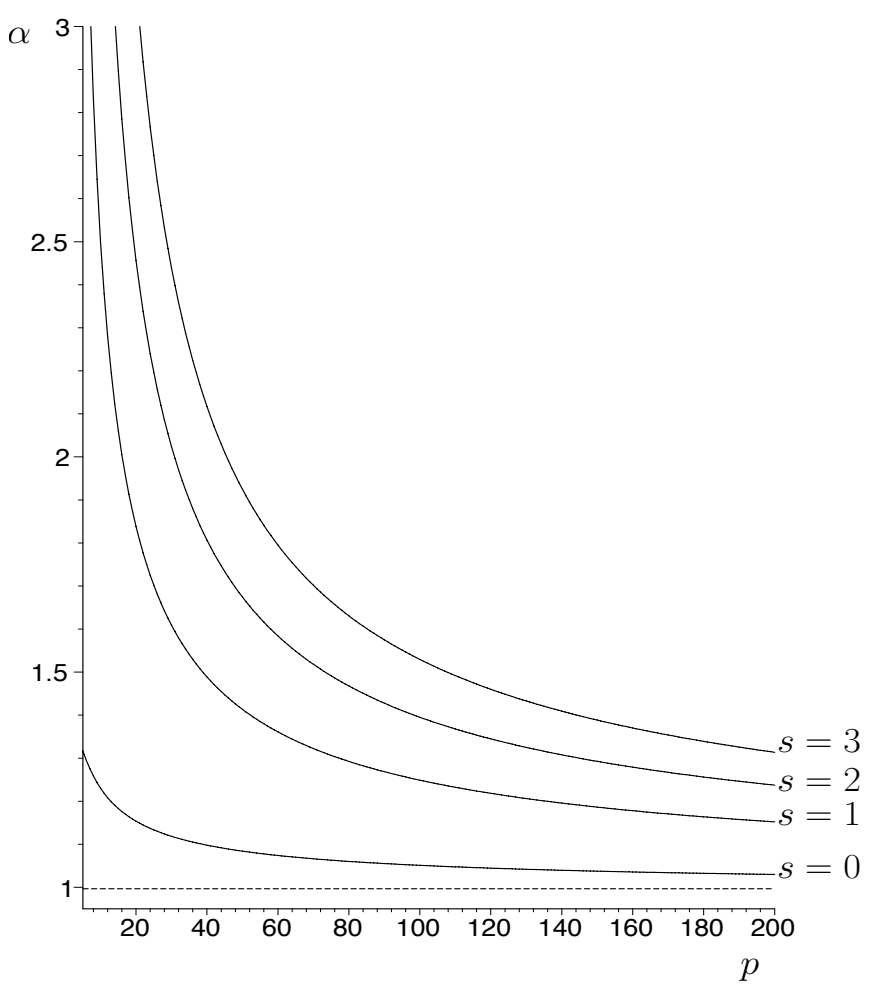

Fig. (3). Dispersion curves for a disk made of brass $(v=0.34)$. The dashed line represents the speed of the straight-edge wave.

Finally, the profiles of the disk are shown on Fig. (4) at $p=200$ for $s=0,1,2$; there the distance was normalized with respect to the radius of the disk and the elevation at the edge of the disk was taken as half the radius (exaggerated scale). For $s=0$ (no nodal circle), the amplitude decays rapidly away from the edge, and is almost zero at $0.9 R$. For $s=1,2$, the amplitude of the wave reaches a maximum which is greater than its value at the edge (amplification); it is also less localized than for $s=0$, being almost zero at about $0.8 R$. Seen from above, the case $s=2$ (two nodal circles) will display one shaded region and one bright region close to the edge, a pattern which is somewhat similar to that recorded by Schedin, Gren, and Rossing [6].

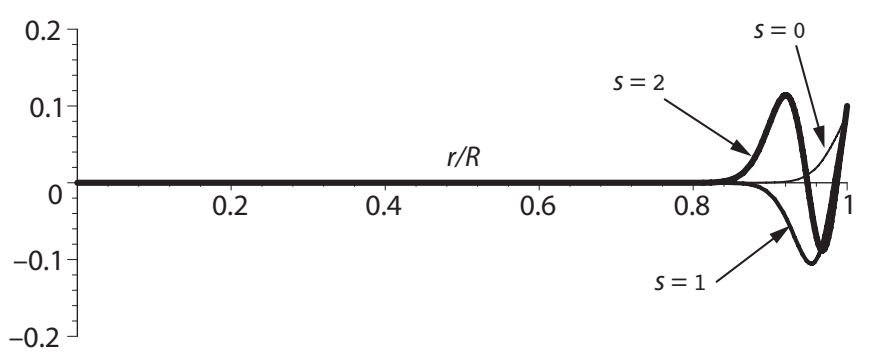

Fig. (4). Profile of a disk made of brass $(v=0.34)$ when $p=200$, $s=0,1,2$.

\section{ACKNOWLEDGEMENTS}

This research was supported by the British Council (UK) and the Ministère des Affaires Etrangères (France).

\section{REFERENCES}

[1] Airey JR. The vibrations of circular plates and their relation to Bessel functions. Proc Phys Soc Lond 1910; 23: 225-32. 
[2] Touzé C, Thomas O, Chaigne A. Asymmetric non-linear forced vibrations of free-edge circular plates. Part 1: Theory. J Sound Vib 2002; 258: 649-76.

[3] Lord Rayleigh. On waves propagated along the plane surface of an elastic solid. Proc R Soc Lond 1885; 17: 4-11.

[4] Konenkov YK. A Rayleigh-type flexural wave. Sov Phys Acoust 1960; 6: 122-3.

[5] Cerv J. Dispersion of elastic waves and Rayleigh-type waves in a thin disc. Acta Tech C 1988; 1: 89-99.

[6] Schedin S, Gren PO, Rossing TD. Transient wave response of a cymbal using double-pulsed TV holography. J Acoust Soc Am 1998; 103: 1217-20.

[7] Bell JFW, Chen JYF. One dimensional Rayleigh waves on straight and curved edges. Acustica 1984; 56: 77-90.

[8] Zil'bergleit AS, Suslova IB. Contact flexural waves in thin plates. Sov Phys Acoust 1988; 29: 108-11.
[9] Norris AN. Flexural waves on narrow plates. J Acoust Soc Am 2003; 113: 2647-58

[10] Hashimoto Y, Koike Y, Ueha S. Transporting objects without contact using flexural traveling waves. J Acoust Soc Am 1998; 103: 3230-3.

[11] Shaw EAG. On the resonant vibrations of thick Barium Titanate disks J Acoust Soc Am 1956; 28: 38-50.

[12] Mansfield EH. The bending and stretching of plates. Pergamon: Oxford 1964.

[13] Thurston RN, McKenna J. Flexural acoustic waves along the edge of a plate. IEEE Trans Sonics Ultrasonics 1974; su-21: 296-7.

[14] Abramowitz M, Stegun IA. Handbook of mathematical functions. Dover: New York 1965.

[15] Viktorov IA. Rayleigh and Lamb waves. Plenum: New York 1967.

[16] Leissa A. Vibration of plates. Acoustical Society of America, New York, 1993.

[17] Norris AN. Flexural edge waves. J Sound Vib 1994; 171: 571-3. 